\title{
Resolution of disseminated fusariosis in a child with acute leukemia treated with combined antifungal therapy: a case report José-Manuel Vagace*1, Cesar Sanz-Rodriguez ${ }^{2}$, Maria-Soledad Casado1, Nieves Alonso ${ }^{1}$, Manuel Garcia-Dominguez ${ }^{1}$, Francisco Garcia de la Llana ${ }^{3}$, Luis Zarallo ${ }^{4}$, Miguel Fajardo ${ }^{5}$ and Roberto Bajo ${ }^{1}$
}

Address: ${ }^{1}$ Department of Hematology, Complejo Hospitalario Infanta Cristina, Badajoz, Spain, ${ }^{2}$ Department of Clinical Research, Merck Sharp \& Dohme of Spain, Madrid, Spain, ${ }^{3}$ Department of Internal Medicine, Complejo Hospitalario Infanta Cristina, Badajoz, Spain, ${ }^{4}$ Department of Pediatrics, Complejo Hospitalario Infanta Cristina, Badajoz, Spain and ${ }^{5}$ Department of Microbiology, Complejo Hospitalario Infanta Cristina, Badajoz, Spain

Email: José-Manuel Vagace* - jvagacev@aehh.org; Cesar Sanz-Rodriguez - cesar_sanzrodriguez@merck.com; Maria-

Soledad Casado - marisol.casado@ses.juntaex.es; Nieves Alonso - malonsoe@aehh.org; Manuel Garcia-Dominguez - garciadoz@yahoo.es; Francisco Garcia de la Llana - francisco.garcial@ses.juntaex.es; Luis Zarallo - luis.zarallo@ses.juntaex.es;

Miguel Fajardo - miguel.fajardo@ses.juntaex.es; Roberto Bajo - roberto.bajo@ses.juntaex.es

* Corresponding author

Published: 10 May 2007

BMC Infectious Diseases 2007, 7:40 doi:10.1 186/147/-2334-7-40

This article is available from: http://www.biomedcentral.com/I47/-2334/7/40

(C) 2007 Vagace et al; licensee BioMed Central Ltd.

This is an Open Access article distributed under the terms of the Creative Commons Attribution License (http://creativecommons.org/licenses/by/2.0), which permits unrestricted use, distribution, and reproduction in any medium, provided the original work is properly cited.
Received: 19 August 2006

Accepted: 10 May 2007

\begin{abstract}
Background: Fusarium spp. is being isolated with increasing frequency as a pathogen in oncohematologic patients. Caspofungin and amphotericin B have been reported to have synergistic activity against Fusarium spp.

Case presentation: We herein report a case of disseminated fusariosis diagnosed by chest CT scan and positive blood cultures to Fusarium spp. Because the patient's clinical condition deteriorated, CRP levels increased, and blood cultures continued to yield Fusarium spp. despite liposomal amphotericin B monotherapy up to $5 \mathrm{mg} / \mathrm{kg}$ daily, treatment with caspofungin was added. Within 2 weeks of onset of combined antifungal therapy, the chest CT scan demonstrated a progressive resolution of the pulmonary lesions. Upon discontinuation of intravenous antifungals, the patient received suppressive therapy with oral voriconazole. Three months later, a chest CT scan showed no abnormalities. Twenty-five months after discontinuation of all antifungal therapy, the patient remains in complete remission of her neoplastic disease with no signs of clinical activity of the Fusarium infection.
\end{abstract}

Conclusion: This is the first description of successful treatment of disseminated fusariosis in a pediatric patient with acute lymphoblastic leukemia with caspofungin and amphotericin B followed by oral suppressive therapy with voriconazole.

\section{Background}

Fusarium spp. is a saprophytic mould that is ubiquitous in the soil. Invasive fusariosis may follow the inhalation of airborne conidia or the inoculation of conidia through a skin breach associated with indwelling catheters, wounds, burns, or onychomycosis. Deep-seated and disseminated infections caused by Fusarium spp. are being diagnosed with increasing frequency in patients with hematological 
malignancies [1]. The Fusarium species most frequently involved in human infections are Fusarium solani, F. oxysporum, F. verticilloides, and F. moniliforme [1]. Of note, Fusarium species are often confused with Aspergillus spp. as both pathogens have similar histopathologic appearance with septate, dichotomously branching hyphae. Yet, the prognosis of invasive fusariosis is much poorer than for invasive aspergillosis, with very low response rates and most patients with disseminated Fusarium infection dying [1]. We describe here the first case of a favorable response of disseminated fusariosis to combination caspofungin and liposomal amphotericin B followed by oral suppressive therapy with voriconazole in a pediatric patient with acute lymphoblastic leukaemia (ALL).

\section{Case presentation}

A 11-year-old, 50-kg female with a diagnosis of CD10+ ALL was admitted to the hospital with fever and intermittent chest pain. The patient was in complete remission (CR) following SHOP-99 chemotherapy. At the time of hospital admission, she was receiving G-CSF for severe granulocytopenia post-intensification with cytarabine, and had also received broad-spectrum antibiotics and fluconazole prophylaxis during previous episodes of febrile neutropenia.

The physical examination was normal and the patient's general condition good. The blood examination showed 12,500 leukocytes $/ \mathrm{mm}^{3}$ (82\% neutrophils). An urine examination, a chest X-ray, and an abdominal ultrasonography did not reveal abnormal findings. Blood, urine, and stool cultures were also negative. She was initially treated with cefepime and teicoplanin. G-CSF was discontinued, after which the neutrophil count stabilized at around $1,700 / \mathrm{mm}^{3}$. CRP increased steadily up to $5.6 \mathrm{mg} / \mathrm{dL}$ despite broad-spectrum antibiotic therapy. One week later, while continuously febrile, the patient experienced worsening chest pain. A chest CT scan revealed several nodules $1 \mathrm{~cm}$ or smaller in diameter at the apex of both lungs (Figure 1). The Aspergillus galactomannan antigen was negative. Cultures of separate blood samples obtained percutaneously and from the central venous catheter, yielded Fusarium spp. The species of the infecting Fusarium could not be identified and in vitro susceptibility could not be tested. Liposomal amphotericin B (3 mg/kg/ day) was then started. Although the dose of liposomal amphotericin B was increased to $5 \mathrm{mg} / \mathrm{kg}$ daily and the central venous catheter was removed (cultures of the catheter tip were sterile), the patient remained febrile and chest pain continued to worsen over the following 7 days. Blood cultures remained positive for Fusarium spp. and CRP levels increased up to $10.6 \mathrm{mg} / \mathrm{dL}$ (Figure 1). Caspofungin was then initiated (70-mg load followed by $50 \mathrm{mg}$ daily). Fever disappeared within 48 hours of caspofungin onset (Figure 1). Chest pain improved significantly, blood cultures became negative, and CRP levels went down to the $1-2 \mathrm{mg} / \mathrm{dL}$ range over the following days. The patient presented two isolated fever spikes 8 and 12 days after the onset of caspofungin, which corresponded to a phlebitis episode and a limited reaction to L-asparaginase, respectively. Two weeks later, a new chest CT scan demonstrated progressive resolution of the lung nodules (Figure 1). Intravenous antifungals were discontinued and chemotherapy and suppressive therapy with oral voriconazole $200 \mathrm{mg}$ twice daily was started. Three months later, a chest CT scan showed complete resolution of the pulmonary lesions, while the patient was asymptomatic (Figure 1). Thus, voriconazole was discontinued. Twenty-five months after discontinuation of all antifungal therapy, the patient remains healthy in the absence of any symptoms of fungal infection and in CR of her neoplastic disease.

Fusarium resistance to most antifungals and the severe immunosuppression-notably long-lasting, severe neutropenia - in oncohematological patients make Fusarium infections commonly fatal [1]. Despite its low activity against Fusarium [2], amphotericin B remains the drug of choice. Voriconazole is licensed for the treatment of fusariosis based on in vitro data and a series of case reports with a reported response rate of $\sim 40 \%$ [3]. Posaconazole also has potential for therapy of systemic fusariosis [4]. In any case, responses obtained with monotherapies remain too low and unsatisfactory.

We decided to treat our patient with amphotericin B and caspofungin for two reasons: (i) In our view amphotericin $B$ remains the mainstay of therapy for fusariosis in pediatric patients; experience with voriconazole in this patient segment is limited [5-7]; (ii) Concurrent antifungal therapy is now generally considered an alternative way of improving outcome in difficult-to-treat invasive mycoses. In a recent in vitro study amphotericin $B$ and voriconazole rendered mainly additive or subadditive interactions against Fusarium spp. [8]. Yet, the concern remains that combining amphotericin B and azoles may lead to antagonism. In addition to the azole inhibition of the synthesis of amphotericin B's pharmacological target ergosterol, amphotericin B-related damage to the fungal cell membrane may interfere with the influx of azoles [9]. In vitro caspofungin-inactive against Fusarium-showed synergistic or synergistic-to-additive interactions with amphotericin $\mathrm{B}$ for at least half of the Fusarium isolates [10]. By inhibiting cell wall synthesis, caspofungin may presumably enhance the penetration of amphotericin $\mathrm{B}$ [9].

We hypothesize that combination antifungal therapy likely contributed to the sucessful treatment of our patient's severe invasive fusariosis, since clinical improvement only became evident after initiating caspofungin. 


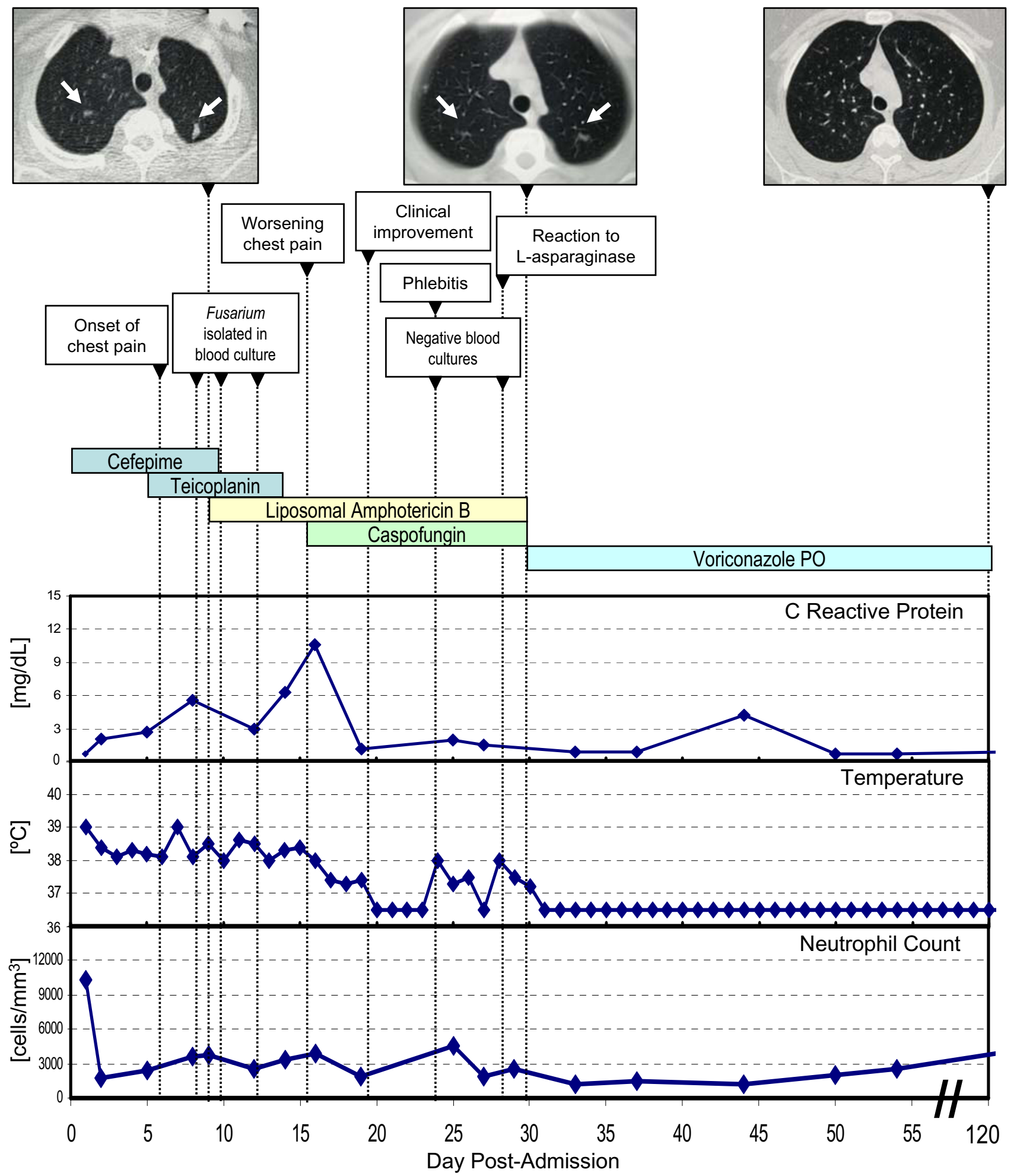

Figure I

Clinical, bio logical, and radiographic evolution and drug therapy. 
Yet, the effect of caspofungin in the successful outcome of our case is not completely clear. The clinical response observed could also be the result of prolonged administration of liposomal amphotericin B and the lack of severe neutropenia. Finally, the administration of suppressive voriconazole and the early re-initiation of chemotherapy allowed by rapid infection control probably also contributed to the good long-term outcome. Of note, there have been a few case reports of response to caspofungin in combination with amphotericin B $[11,12]$ and as monotherapy [13], although in some of these reports there was a clear association of response to recovery from neutropenia.

\section{Conclusion}

We herein report an episode of disseminated fusariosis in a pediatric ALL patient treated successfully with caspofungin and amphotericin B followed by suppressive therapy with voriconazole. While our anecdotal observation is encouraging, the use of combination antifungal therapy is debatable and further in vivo studies are warranted to establish its true role in deep-seated and disseminated Fusarium infections.

\section{Abbreviations \\ CD10+: Cluster differentiation 10-positive}

CT: Computed tomography.

SHOP-99: The standard protocol of the Spanish Society of Pediatric Hematology and Oncology for high risk acute lymphoblastic leukemia, which includes induction (prednisone, daunomycin, vincristine, cyclophosphamide, metothrexate, L-asparaginase), consolidation (metothrexate, cytarabine, mercaptopurine), and intensification (adriamycin, vincristine, dexamethasone, cyclophosphamide, metothrexate, cytarabine, L-asparaginase) chemotherapy.

G-CSF: Granulocyte-colony stimulating factor.

CRP: $\mathrm{C}$ reactive protein.

\section{Competing interests}

C. Sanz-Rodriguez is an employee of Merck Sharp \& Dohme de España, S.A.. The remaining authors declare that they have no competing interests.

\section{Authors' contributions}

J-M. Vagace, M-S. Casado, N. Alonso, M. GarciaDominguez, F. Garcia de la Llana, L. Zarallo, M. Fajardo, and R. Bajo participated actively in the diagnosis, care, and follow-up of the patient.
$\mathrm{J}-\mathrm{M}$. Vagace obtained consent from the patient's parents for this case report, reviewed the literature, provided clinical details, and drafted the manuscript.

C. Sanz-Rodriguez reviewed the literature and gave helpful comments regarding the scientific content of the manuscript.

All authors read and approved the final manuscript.

\section{Acknowledgements}

Written consent was obtained from the patient's parents for publication of study. There was no financial support for the preparation of this manuscript.

\section{References}

I. Boutati El, Anaissie Ej: Fusarium, a significant emerging pathogen in patients with hematologic malignancy: ten years' experience at a cancer center and implications for management. Blood 1997, 90:999-1008.

2. Anaissie EJ, Hachem R, Legrand C, Legenne P, Nelson P, Bodey GP: Lack of activity of amphotericin $B$ in systemic murine fusarial infection. J Infect Dis 1992, 165: I I55-I I57.

3. The European Agency for the Evaluation of Medicinal Products: Vfend (voriconazole), Scientific Discussion, European Public Assessment Report. London 2005 [http://www.emea.eu.int/humandocs/Humans/EPAR/vfend/ vfend.htm]. Accessed October 4, 2005

4. Raad II, Hachem RY, Herbrecht R, Graybill JR, Hare R, Corcoran R, Kontoyiannis DP: Posaconazole as salvage treatment for invasive fusariosis in patients with underlying hematologic malignancy and other conditions. Clin Infect Dis 2006, 42:1398-403.

5. Baden LR, Katz JT, Fishman JA, Koziol C, Del Vecchio A, Doran M, Rubin RH: Salvage therapy with voriconazole for invasive fungal infections in patients failing or intolerant to standard antifungal therapy. Transplantation 2003, 76:1632-1637.

6. Guzman-Cottrill JA, Zheng X, Chadwick EG: Fusarium solani endocarditis successfully treated with liposomal amphotericin B and voriconazole. Pediatr Infect Dis J 2004, 23: 1059-106I.

7. Rodriguez CA, Lujan-Zilbermann J, Woodard P, Andreansky M, Adderson EE: Successful treatment of disseminated fusariosis. Bone Marrow Transplant 2003, 3 I:4I I-4I 2.

8. Ortoneda M, Capilla J, Javier Pastor F, Pujol I, Guarro J: In vitro interactions of licensed and novel antifungal drugs against Fusarium spp. Diagn Microbiol Infect Dis 2004, 48:69-7I.

9. Cuenca-Estrella $\mathrm{M}$ : Combinations of antifungal agents in therapy - what value are they? J Antimicrob Chemother 2004, 54:854-869.

10. Arikan S, Lozano-Chiu M, Paetznick $\mathrm{V}, \operatorname{Rex} \mathrm{JH}$ : In vitro synergy of caspofungin and amphotericin $B$ against Aspergillus and Fusarium spp. Antimicrob Agents Chemother 2002, 46:245-247.

II. Makowsky MJ, Warkentin DI, Savoie ML: Caspofungin and amphotericin B for disseminated Fusarium verticillioides in leukemia. Ann Pharmacother 2005, 39:1365-1366.

12. Fatoye A, Bow EJ, Loewen R: Amphotericin B lipid complex and caspofungin as combination therapy for disseminated fusariosis in a patient with acute myeloid leukemia [abstract]. In Proceedings of the 13th Congress of the International Society for Human and Animal Mycology: 25-29 May 2003; San Antonio, TX the International Society for Human and Animal Mycology; 2003:392.

13. Apostolidis J, Bouzani M, Platsouka E, Belasiotou H, Stamouli M, Harhalakis N, Boutati El, Paniara O, Nikiforakis E: Resolution of fungemia due to Fusarium species in a patient with acute leukemia treated with caspofungin. Clin Infect Dis 2003, 36: I349-1350.

\section{Pre-publication history}

The pre-publication history for this paper can be accessed here:

http://www.biomedcentral.com/1471-2334/7/40/prepub 